\title{
Stem Cells for Retinal Replacement Therapy
}

\author{
Jeffrey H. Stern • Sally Temple
}

Published online: 27 September 2011

(C) The American Society for Experimental NeuroTherapeutics, Inc. 2011

\begin{abstract}
Retinal degenerative disease has limited therapeutic options and the possibility of stem cell-mediated regenerative treatments is being actively explored for these blinding retinal conditions. The relative accessibility of this central nervous system tissue and the ability to visually monitor changes after transplantation make the retina and adjacent retinal pigment epithelium prime targets for pioneering stem cell therapeutics. Prior work conducted for several decades indicated the promise of cell transplantation for retinal disease, and new strategies that combine these established surgical approaches with stem cell-derived donor cells is ongoing. A variety of tissue-specific and pluripotent-derived donor cells are being advanced to replace lost or damaged retinal cells and/or to slow the disease processes by providing neuroprotective factors, with the ultimate aim of long-term improvement in visual function. Clinical trials are in the early stages, and data on safety and efficacy are widely anticipated. Positive outcomes from these stem cell-based clinical studies would radically change the way that blinding disorders are approached in the clinic.
\end{abstract}

Keywords Retina - Stem cell · Replacement therapy · Transplant $\cdot$ Macular degeneration $\cdot$ Retinitis pigmentosa

Electronic supplementary material The online version of this article (doi:10.1007/s13311-011-0077-6) contains supplementary material, which is available to authorized users.

J. H. Stern $(\bowtie) \cdot$ S. Temple

Neural Stem Cell Institute,

Rensselaer, NY 12144, USA

e-mail: retina@nycap.rr.com

\section{Introduction}

In early neural development, the retina emerges as an accessible and highly structured part of the central nervous system (CNS). Vision is initiated by the photoreceptor cell layer of the neural retina and is then processed by downstream neurons in the bipolar, ganglion, horizontal, and amacrine cell layers. Glial Mueller cells span the neural retina and provide support to it. The retinal pigment epithelium (RPE) is a separate layer of the retina, which is adjacent to the photoreceptor cell layer. The RPE and photoreceptor cells both participate in the visual pigment cycle.

For the past few decades, transplantation to replace the compromised photoreceptor cell or RPE retinal layers has been found to improve visual function. These pioneering studies have been advanced using stem cells to generate large quantities of cells suitable for retinal replacement. The well-defined two-dimensional photoreceptor cell and RPE retinal layers structure are an ideal target for replacement therapy. Surgical accessibility, sensitive measurements of structure and function, the ability to ablate or remove failed grafts, and a great unmet medical need all combine to make the retina a point of convergence for translating stem cell research into clinical therapy.

\section{Retinal Replacement Therapy}

Early transplants of mammalian retina in 1959 showed that fetal retina remains viable for months after transplantation into the adult eye [1]. When embryonic fetal aggregates or sheets are transplanted into an adult 
rodent retina, cellular rosettes displaying retinal characteristics form with partial integration into the host [2]. Integration increases when the younger donor or host tissue is used [3], and also when the host retina is injured [4]. Significantly, transplantation of fetal retina into diseased host retina improves behavioral and objective measures of vision in those animals [5].

Retinal progenitor cells (RPCs), considering the active cellular component of fetal retinal transplants, were purified from green fluorescent protein transgenic mice and transplanted into the degenerating retina of a mature mouse model. The transplanted RPCs developed into neurons, including presumptive photoreceptor cells expressing rhodopsin, opsin, and recoverin. The host showed rescue of the outer retina layer cells with integration of donor cells occurring in multiple retinal layers. The greatest concentration of integration in the outer retina, and most importantly, recipient mice, demonstrated an improved response to light [6]. Coating fetal retinal sheets with microspheres, containing brain-derived neurotrophic factor as a mitogen for RPCs, improves the functional efficacy of fetal aggregate grafts [7]. Subsequently, sheets of fetal retina transplanted into rats showed appropriate integration and synaptic connectivity along with visual restoration [8]. These studies were then applied to patients and transplantation of human fetal retinal sheets was found to improve the vision of patients transiently for a period of 3 to 13 months [9]. Clinical trials transplanting sheets of fetal donor retina and RPE cells into patients are ongoing in the United States (http://clinicaltrials.gov/ct2/show/NCT00345917; http://clinicaltrials.gov/ct2/show/NCT00346060 [10]) and Germany (clinicaltrials.gov NCT00401713, [11]). These trials demonstrate that human retinal transplantation is feasible, and they hold the promise of visual recovery for otherwise hopeless retinal disease [12]. A limitation of these studies, however, is the difficulty of obtaining sufficient human fetal retina donor tissue for treatment of large clinical populations.

Retinal replacement therapy can be accelerated by the use of stem cells that self-renew and differentiate into large populations of progeny suitable for transplantation. Active research aims to determine which stem cells are best suited to replace the retina and to identify the retinal layers and diseases most amenable to stem cell replacement therapy. Ongoing animal and early stage human studies are targeting the photoreceptor cell layer and the RPE layer. These layers appose each other to form the surgically accessible subretinal potential space where cells can be readily placed. Important diseases including retinitis pigmentosa (RP) and age-related macular degeneration (AMD) arise in these layers. Several stem cell types are currently under investigation to repopulate the RPE and photoreceptor cell retinal layers.

Although the neural retina and RPE are distinct anatomically, in development they share a closely related neuroectodermal origin. As the optic cup forms, RPE differentiation first begins at 4 to 6 weeks of gestation in humans, and then differentiation of neural retina follows [13]. In adults, the neural retina and RPE have overlapping regenerative capacity after injury (e.g., in amphibians, injury can activate both retinal and RPE progenitor cells to mediate regeneration and repair). Limited damage of amphibian retina activates progenitor cells within the neural retina, presumably retina stem cells (RSCs), to mediate regeneration and repair (for more detail see Reh and Levine [14]), whereas extensive damage that destroys most of the neural retina results in the activation of a progenitor cell population within the RPE layer, presumably retinal pigment epithelial stem cells (RPESCs), to regenerate both neural retina and RPE [15]. This plasticity seen in lower animals is inhibited in higher animals in which RSCs and RPESCs remain dormant after injury, and (as in most parts of the mammalian CNS) little regeneration or repair occurs.

\section{RSCs}

RSCs have been isolated from the marginal periphery of the neurosensory mammalian retina [16, 17], although questions have been raised regarding the true origin of these cells and the ability to self-renew [18]. More recently, RSCs have been isolated from the posterior neural retina that demonstrate self-renewal and differentiated progeny (2009 United States Patent No. $7,514,259$ B2, available at: www.uspto.gov). Of the multiple types of RSCs and RPCs reported, retinal glial Mueller cells are the most studied late progenitors that retain competency to produce neuronal lineages including photoreceptor cells [19]. Mueller glial cells actively regenerate damaged retina in lower animals and can be similarly activated in mice by application of growth factors [20]. Although the Mueller cells reside in the retina and have properties of RSCs, they also produce all major neural lineages, in which there are multipotent neural stem cells [21].

Retinal stem cells can be expanded though multiple passages to generate large numbers of cells. Expanded populations can then be differentiated into the major neural retinal cell types, including photoreceptor cells. When transplanted, RSCs incorporate into the neural retina and rescue vision in animal models (for more detail see Lamba et al. [22] and Wallace [23]. As with 
RPCs, RSCs transplanted into the subretinal space of young mice survive, migrate, integrate, and differentiate into appropriate cell types, including photoreceptor cells [24]. In healthy adult recipients, however, transplanted RSCs preferentially express ganglion cell or glial markers rather than the photoreceptor cell type needed for replacement of the outer retina [25]. Furthermore, as also found with transplantation of retinal aggregates, RSCs from younger donors integrate more appropriately than those derived from older donors, and more extensive integration occurs when the host retina is injured or diseased [26, 27]. When retinal progenitor cell populations containing RSCs are isolated at the developmental age that normally generates photoreceptor cells, transplantation was found to generate predominately photoreceptor cells in the adult mouse retina [28].

In addition to primary RSCs derived from retinal tissue, RSCs can be derived from embryonic stem cells (ESCs) $[29,30]$. Embryonic stem cells can be expanded extensively to generate the large numbers of cells needed for commercial development. Embryonic stem cell-derived RSCs differentiate into all the major neural retina phenotypes and will even remarkably form three-dimensional retinal structures in vitro [31]. Retina progenitor cells derived from human ESCs transplanted into the mouse eye migrate into appropriate retinal layers and express differentiated markers, including those specifying photoreceptor cells, and they restore visual responses in a mouse model of outer retinal disease [32].

The committed fate and lineage choice of RSCs is advantageous for their clinical use in retinal replacement therapy and progress to control the development of primary RSCs and ESCs-RSCs to generate progeny appropriate for replacement therapy is advancing at a rapid pace. Challenges such as obtaining sufficient donor source material and control of immune reactivity can be addressed; organized donor banks or improved expansion can generate large numbers of cells, whereas immune suppression or immune type matching can improve immune compatibility. Currently, substantial interest and investment in RSCs and ESCs-RSCs for replacement therapy of photoreceptor cell layer disease has not prompted the initiation of clinical studies transplanting RSCs.

\section{RPESCs}

The RPE is one of the first neural cell types in the CNS to fully differentiate. Terminal differentiation begins at 4 to 6 weeks of gestation in humans, with such earlydifferentiated RPE cells remaining dormant throughout life [13]. After differentiation at an early gestational age, these differentiated RPE cells do not divide, and thus, such RPE cells present in adult humans are the same cells as present in the 4- to 6-week human embryo. In our laboratory, quiescent adult RPE have been activated to self-renew when cultured under appropriate proliferative conditions (unpublished data). In addition to displaying the defining property of stem cells (i.e., self-renewal), when they are cultured under defined differentiation protocols, these RPESCs differentiate into a variety of progeny, including RPE and mesenchymal lineages. RPESCs are not pluripotent and do not produce endodermal lineages, but they are multipotent-producing ectodermal and mesenchymal lineages, including RPE (unpublished data). Human RPESCs are a promising potential stem cell source for allogenic or autologous RPE replacement therapy that has not yet been evaluated in animal models or patients.

The fact that RPE cells remain dormant from early on in development indicates a close lineage relationship between adult RPE and early embryonic cells. It is not surprising, in this context, that ESCs readily differentiate into RPE [33, 34]. ESCs expand extensively to produce large quantities of cells that can differentiate into all progeny types, and thus there is great interest in developing ESCs to treat diverse diseases. ESCs propensity to produce RPE cells under standard culture conditions makes the RPE a good target for ESC-sourced replacement therapy. ESC-RPEs injected into animal models differentiate and integrate into the host RPE layer to rescue photoreceptor cell loss in animal models [35, 36]. Tumor formation was not observed for more than 220 days after injection of ESC-RPEs into a rat model [36]. Concern regarding tumorigenicity remains, however, because ESC tumors can be more virulent in homologous than in xenografted hosts, as is true for the production of inappropriate progeny types (e.g., non-neural cells after placement in the CNS) $[37,38]$. Immune rejection with allogenic ESC-RPE transplants can be controlled by immune suppression of the host. The Food and Drug Administration has approved commercial study of ESCRPEs transplantation for the treatment of retinal patients, and pending human trials have been announced by Advanced Cell Therapeutics (www.advancedcell.com).

\section{Neural Stem Cells}

Another promising stem cell source for retinal replacement therapy is neural stem cells (NSCs) derived from the committed central nervous tissue. Although NSC integration into the retina is limited, transplanted NSCs expand and rescue both photoreceptor cells and vision in animal models of outer retinal disease. Secretion of 
growth factors and/or metabolic processing of phototransduction byproducts by NSCs are thought to mediate this rescue.

Neural stem cells are found in many regions of the embryonic nervous system, including the retina [21], with the richest source for transplantation being forebrain-derived NSCs (for more detail see Temple [39]). Green fluorescent protein-expressing forebrain NSCs survive and display limited morphologic characteristic of retinal neurons with limited integration after transplantation into the mouse retina. Some of the transplanted NSCs integrate into inappropriate retinal layers with the age of the host having an important role in determining NSC fate [40-42], whereas other NSCs do not integrate but survive in the subretinal space with limited expression of retinal cell phenotypes [40]. As with RPCs, integration increases when NSCs are transplanted into young or injured host retina [43, 44], and at the other extreme, NSCs transplanted into healthy adult monkey show little migration or integration, forming a monolayer of stable NSCs [45]. Integration may not be necessary to rescue photoreceptor cell loss; however, NSCs derived from committed CNS tissue rescue photoreceptor cells in animal models of retinal disease presumably by release of growth factors and/or phagocytosis of photoreceptor cell outer segments shed during the early steps of vision [46, 47].

Primary NSCs derived from fetal donor brains can be expanded extensively [39], and efforts to use these for clinical replacement therapy are in the process. Companies including Stem Cells, Inc., CellCure, and others have announced significant investments in preclinical studies of NSCs as potential replacement therapies for human retinal degenerations. Clinical trials using primary NSCs as therapy for retinal disease have not begun.

In addition to primary source-derived NSCs, such as those harvested from fetal donor brains, large numbers of NSCs can be produced by differentiation of ESCs. When transplanted into the retina, ESC-derived NSCs show limited incorporation and differentiation into photoreceptor cell fates. Importantly, functional rescue of the outer retina in an animal model is observed after transplantation of ESC-NSCs [48-50]. Teratomas were not observed after transplantation of ESC-NSCs, although tumors were observed with related neurally selected ESCs [51]. Directing ESCs toward NSCs that express retinal markers prior to transplantation improves integration and increases the number of photoreceptor cell progeny, whereas tumor formation using this protocol was not observed for as much as 6 weeks [32]. Controlled clinical trials using ESC-NSCs to treat retinal disease have not begun.

\section{Non-Neural Stem Cells}

Another stem cell-based strategy for retinal replacement therapy is the transplantation of non-neural stem cells, such as mesenchymal stem cells (MSCs), bone-marrow stem cells (BMSCs), or umbilical cord blood stem cells (UCSCs). For stem cells with endothelial potential, retinal revascularization can be observed directly through the pupil, making the retina a good model system for studying stem cell-mediated CNS revascularization in general. Restoration of vasculature located on the inner retinal surface by BMSCs is a rational approach toward rescue of retinal neurons lost in retinal vascular disorders, such as diabetic retinopathy [52-54]. Injection of BMSCs into the eye improves retinal circulation, enhances survival of outer retinal neurons, and rescues vision in both ischemic and nonischemic mouse models of retinal degeneration $[53,55]$. These findings open a new paradigm for the relationship between vasculature and the neural retina in which neurotrophic effects secondary to improved circulation after BMSC transplantation benefits both ischemic and nonischemic retinal degenerations [56, 57].

In addition to improving retinal circulation, transplanted BMSCs integrate into the neural retina and undergo limited retinal differentiation that corresponds with the rescue of vision and outer retina in animal models. The finding of MSCderived cells that express some of the features of neural retina cells progeny after transplantation raises some possibility that aspects of cell replacement can occur and play a role in BMSC-mediated rescue of vision [58, 59]. Some studies indicate that rescue is due to retinal integration and neural differentiation of BMSCs to replace outer retinal cells [6062], whereas others indicate that non-neural cells, such as MSC mediate rescue of the photoreceptor cell layer is due to improved circulation and secretion of growth factors essential for photoreceptor cell survival [59, 63].

Although the distinction between rescue of vision via angiogenesis, neurotrophic action, and/or cellular replacement is not fully resolved, there is agreement that nonneural stem cell transplantation prevents vision loss in animal models, suggesting that transplantation of such cells may benefit retinal patients. Pre-clinical studies of MSCs to treat both non-ischemic retinal diseases, such as AMD or RP and ischemic ones, such as diabetic retinopathy have been announced on the websites of companies, including Athersys, Mesoblast, Centocor, and others. A phase I clinical study was completed by the Stem Cell Organization of Centocor, Inc. and J $+\mathrm{J}$ Biotechnology, following patients for more than 1 year after subretinal injection of UCSC doses ranging from 47,500 to 470,000 cells (http://clinicaltrials.gov/ct2/show/NCT00458575? term $=$ NCT00458575\&rank=1 clinicaltrials.gov). Immune 
rejection of the UCSC grafts was limited and complications related to surgical technique were reported in this carefully constructed and pioneering step forward in the retinal stem cell field. A clinical study in San Paulo, Brazil reported injection of autologous BMSCs into the eyes of RP patients to be safe (http://clinicaltrials.gov/ ct $2 /$ show/NCT01068561?term $=$ NCT01068561\&rank=1). The use of MSCs is clouded, however, by an active unregulated medical tourism trade particularly focused on using autologous BMSCs to treat a wide spectrum of retinal disease. Evaluation of these uncontrolled treatments is beyond the scope of this review, because there is very limited information available regarding the outcomes or rationale. At this time, results from ongoing sciencebased controlled clinical studies of MSC replacement therapy in retinal patients are eagerly awaited.

\section{Pluripotent Stem Cells}

The advent of ESCs and induced pluripotent stem cells (iPSCs) raises the possibility that injecting progeny derived from pluripotent cells into the retinal microenvironment will induce their differentiation into appropriate retinal progeny. Pluripotent cells have a propensity to differentiate into retinal progeny and spontaneously form RPE progeny in cultures or even a primitive eyecup if maintained long enough under appropriate conditions [31].

Early studies explored whether direct transplantation of ESCs into the eye would be beneficial, but this approach was quickly discounted as results showed development of tumors containing progeny from all germ lines. Teratomas form even after transplantation of ESCs that have undergone neural selection [51]. Although pre-transplantation differentiation of ESC into RSCs, NSCs, or RPE decreases tumor formation, tumorigenicity remains a challenge for the safe clinical use of ESCs [64]. Maximizing the purity of the differentiated progeny to be transplanted to minimize tumor risk from residual source ESCs appears to be sufficient in animal model experiments. Animal models, however, may not be ideal for measuring tumorigenicity, because immune surveillance is heightened when transplanting across species. Nevertheless, a small risk of tumor formation is tolerable in selected clinical situations in which a potential large benefit outweighs the small risk that a tumor forms. The ability to detect and ablate or otherwise remove tumors is an important advantage of the retina as compared to other areas of the CNS considered for stem cell transplants, and there is steady progress to resolve tumor-related limitations to the use of pluripotent cells for retinal replacement.
Although pluripotent cells are naive in terms of immune reactivity and only express some human leukocyte antigen types [65], transplantation into retina results in immune reaction to differentiated progeny. The immune privilege of retina and reduced immunogenicity of undifferentiated ESCs may favor immune compatibility, but these conditions are not sufficient to prevent rejection, and immune suppression after transplantation of allogenic ESC-derived cells into the retina is needed to prevent rejection of transplanted tissue [36]. One way to avoid immune rejection is by the use of pluripotent stem cells created by transforming a patient's somatic cells into induced pluripotent stem cells (iPSCs) [66].

Induced pluripotent stem cells, created by inserting 4 genes into a patient's skin cells, share properties of ESCs, but are immune-matched to the donor. iPSCs can be directed to a variety of lineages, including RSCs and RPESCs. iPSC-derived RPE-like cells (iPSC-RPEs) [67-70] transplanted into the retina show incorporation into the photoreceptor layer with rescue of vision in an animal model [71]. RSCs have also been derived from iPSCs by selecting retinal rosettes [68] or by directing differentiation with small molecules [72]. Although the process for deriving pure populations of retinal precursors from iPSCs is less efficient than for ESCs, culture protocols to differentiate iPSC-RSCs into photoreceptor cell precursors are sufficiently developed so that such progeny integrate into mouse retina and differentiate into both rod and cone photoreceptor cells [71]. Transplantation of iPSCs differentiated into functional iPS-RPE into the RCS rat retina resulted in the maintenance of longterm visual function, even after the xenografted cells were eventually lost to immune rejection, raising the possibility that a secondary protective host cellular response rescued photoreceptor cells [73]. iPSC are a promising source for retinal replacement therapy, although with significant remaining challenges to their clinical use. Oncogenederived iPSC tumorigenicity needs to be overcome prior to the use of iPSC-derived source material in human transplants [74]. Regulatory approvals will be complex due to the extensive manipulation, whether with gene insertion or application of other factors. iPSC-based retinal replacement also faces challenges raised by residual epigenetic factors that cause variation in their differentiation profile, making each clone unique to the individual patient source, as well as by the risk of an increased rate of somatic mutations in cancer-associated genes [75].

As with iPSCs, parthogenic stem cells (phSCs) are pluripotent stem cells that can be harvested from a donor, expanded, and then differentiated to generate progeny for retinal replacement that are immune compatible with the 
donor source. The manipulation of donor cells needed to make phSCs also raise the risk for abnormalities in progeny and epigenetic factors (such as the methylation state), each contributing to variations in differentiation protocol outcome. Protocols have been established to generate NSCs and RPEs from phSC [76]. The results of phSCs transplanted into the retina have not been published. Understanding the challenges to the use of phSCs for retinal replacement, the International Stem Cell Corporation is actively developing phSCs for retinal replacement (www.internationalstemcell.com).

\section{Other Uses of Stem Cells for Retinal Therapy}

Replacing damaged cells with normal stem cell progeny is 1 therapeutic application of stem cells. These remarkable cells also generate pathologic progeny useful for studying disease mechanisms, drug discovery, and targeted drug delivery. For example, our laboratory is developing a stem cell-based disease model for dry AMD. The hallmark of dry AMD is proteinaceous extracellular deposits termed drusen, which are formed by diseased RPE cells. RPESCs can be driven to progeny that express drusen proteins in culture. The process generating these pathologic RPESC progeny serves as an in vitro model for dry AMD pathogenesis. This "disease in a dish" model is used to study the mechanism of drusen formation, to screen for drug candidates that inhibit drusen formation, and to test the toxicity of these drug candidates (unpublished data). iPSCs have been used to generate "disease in a dish" models expressing donorspecific genotypes. Disease-specific lines of iPSCs have tremendous potential as drug screening platforms and as in vitro models for efficacy testing of gene therapies and drugs [77]. iPSC lines generated from patients with retinal degeneration can be used for such follow-up studies.

Stem cells can be modified to generate in vitro models and also to create drug delivery devices. Factor-producing cell lines encapsulated within a device surgically inserted into the eye are used in a clinical trial by Neuratech (http:// clinicaltrials.gov/ct2/show/NCT00447993?term $=\mathrm{NC}$ T00447993\&rank=1). In addition, stem cells transplanted into the retina that are thought to rescue vision by delivery of trophic factors can be modified prior to transplant to optimize targeted delivery of therapeutic factors to the outer retina.

\section{Conclusion}

Stem cell sources in preclinical studies for retinal replacement therapy include RSCs, RPESCs, ESC-RPEs, iPSCRPEs, phSC-RPEs, NSCs, ESC-NSCs, BMSCs, and UCSCs (Table 1). All the retinal stem cell types (except RPESCs, which have not been injected into animal eyes) demonstrate photoreceptor cell layer rescue, as well as visual improvement in animal models of retinal disease. Each stem cell source holds promise and provides fresh hope for otherwise devastating blinding retinal disease, whereas each has unique advantages and hurdles yet to be overcome. Clinical trials have been initiated for UCSCs and BMSCs, and have been approved for ESC-RPEs. Rapid progress of this research raises hope that the "right" cells are within reach to make stem cell replacement therapy a reality to provide durable visual improvement for retinal patients with otherwise untreatable blinding conditions.

Table 1 Types of Stem Cells for Retinal Replacement Therapy

\begin{tabular}{llllll}
\hline Stem cell & Rescue vision & Immune compatible & Tumor formation & Expansion & Source \\
\hline RSC & Yes & No & No & Moderate & Cadaver \\
RPESC & Unknown & Yes for self & No & Moderate & Self, donor or cadaver RPE \\
NSC & Yes & No & No & Moderate & Cadaver \\
ESC-RPE & Yes & No & Possible & Extensive & Embryo \\
ESC-NSC & Yes & No & Possible & Extensive & Embryo \\
iPSC-RPE & Yes & Yes for self & Unknown & Extensive & Self, donor or cadaver \\
phSC-RPE & Yes & Yes for self & No & Self, donor or cadaver \\
BMSC & Yes & Yes for self & No & Moderate & Self, donor or cadaver \\
UCSC & Yes & No & Moderate & Umbilical Cord \\
\hline
\end{tabular}

$\mathrm{BMSC}=$ bone marrow stem cell; ESC-NSC = embryonic stem cell-derived neural stem cell; ESC-RPE = embryonic stem cell-derived retinal pigment epithelium; iPSC-RPE $=$ induced pluripotent stem cell-derived retinal pigment epithelium; NSC $=$ neural stem cell; phSC-RPE $=$ parthoegnic stem cell-derived retinal pigment epithelium; RPE $=$ retinal pigment epithelium; RPESC $=$ retina pigment epithelial stem cell; $\mathrm{RSC}=$ retinal stem cell; UCSC $=$ umbilical cord stem cell 
Acknowledgments We thank the Regenerative Research Foundation for continued support.

Full conflict of interest disclosure is available in the Electronic Supplementary Material for this article.

\section{References}

1. Royo PE, aQ, WB. Retinal transplantation from fetal to maternal mammalian eye. Growth 1959;23:313-316.

2. del Cerro M, Gash D, Rao G, Notter M, Wiegand S, Gupta M. Intraocular retinal transplants. Invest Ophthalmol Vis Sci 1985;26:1182-1185.

3. Aramant R, Seiler M, Turner JE. Donor age influences on the success of retinal grafts to adult rat retina. Invest Ophthalmol Vis Sci 1988;29:498-503.

4. Turner JE, et al. Embryonic retinal grafts transplanted into the lesioned adult rat retina. Prog Brain Res, 1988. 78: p. 131-9.

5. del Cerro M, IJ, Bowen G, Lazar E, del Cerro C. Intraretinal grafting restores visual function in light-blinded rats. Neuroreports 1991;2:501-544.

6. Klassen $\mathrm{HJ}$, et al. Multipotent retinal progenitors express developmental markers, differentiate into retinal neurons, and preserve light-mediated behavior. Invest Ophthalmol Vis Sci 2004; 45:4167-4173.

7. Seiler MJ, et al. BDNF-treated retinal progenitor sheets transplanted to degenerate rats: improved restoration of visual function. Exp Eye Res 2008;86:92-104.

8. Seiler MJ, et al. Visual restoration and transplant connectivity in degenerate rats implanted with retinal progenitor sheets. Eur J Neurosci 2010;31:508-520.

9. Humayun MS, et al. Human neural retinal transplantation. Invest Ophthalmol Vis Sci 2000;41:3100-3106.

10. Radtke ND, et al. Vision improvement in retinal degeneration patients by implantation of retina together with retinal pigment epithelium. Am J Ophthalmol 2008;146:172-182.

11. Falkner-Radler CI, et al. Human retinal pigment epithelium (RPE) transplantation: outcome after autologous RPE-choroid sheet and RPE cell-suspension in a randomised clinical study. $\mathrm{Br} \mathrm{J}$ Ophthalmol 2011;95:370-375.

12. Zarbin MA. Retinal pigment epithelium-retina transplantation for retinal degenerative disease. Am J Ophthalmol 2008;146:151-153.

13. Mann I. Development of the Eye. 1929.

14. Reh TA, Levine EM. Multipotential stem cells and progenitors in the vertebrate retina. J Neurobiol 1998;36:206-220.

15. Stone LS. Neural retina degeneration followed by regeneration from surviving retinal pigment cells in grafted adult salamander eyes. Anat Rec 1950;106:89-109.

16. Tropepe V, et al. Retinal stem cells in the adult mammalian eye. Science 2000;287:2032-2036.

17. Ahmad I, Tang L, Pham H. Identification of neural progenitors in the adult mammalian eye. Biochem Biophys Res Commun 2000;270:517-521.

18. Cicero SA, et al. Cells previously identified as retinal stem cells are pigmented ciliary epithelial cells. Proc Natl Acad Sci U S A 2009;106:6685-6690.

19. Moshiri A, Reh TA. Persistent progenitors at the retinal margin of ptc $+/-$ mice. J Neurosci 2004;24:229-237.

20. Karl MO, et al. Stimulation of neural regeneration in the mouse retina. Proc Natl Acad Sci U S A 2008;105:19508-19513.

21. Das AV, et al. Neural stem cell properties of Muller glia in the mammalian retina: regulation by Notch and Wnt signaling. Dev Biol 2006;299:283-302.
22. Lamba D, Karl M, Reh T. Neural regeneration and cell replacement: a view from the eye. Cell Stem Cell 2008;2:538-549.

23. Wallace VA. Concise review: making a retina-from the building blocks to clinical applications. Stem Cells 2011;29:412417.

24. Coles BL, et al. Facile isolation and the characterization of human retinal stem cells. Proc Natl Acad Sci U S A 2004;101:1577215777.

25. Canola K, et al. Retinal stem cells transplanted into models of late stages of retinitis pigmentosa preferentially adopt a glial or a retinal ganglion cell fate. Invest Ophthalmol Vis Sci 2007;48:446454.

26. Chacko DM, et al. Transplantation of ocular stem cells: the role of injury in incorporation and differentiation of grafted cells in the retina. Vision Res 2003;43:937-946.

27. Klassen H, Sakaguchi DS, Young MJ. Stem cells and retinal repair. Prog Retin Eye Res 2004;23:149-181.

28. MacLaren RE, et al. Retinal repair by transplantation of photoreceptor precursors. Nature 2006;444:203-207.

29. Zhao X, Liu J, Ahmad I. Differentiation of embryonic stem cells into retinal neurons. Biochem Biophys Res Commun 2002;297:177-184.

30. Ikeda $\mathrm{H}$, et al. Generation of $\mathrm{Rx}+/ \mathrm{Pax} 6+$ neural retinal precursors from embryonic stem cells. Proc Natl Acad Sci U S A 2005;102:11331-11336.

31. Eiraku M, et al. Self-organizing optic-cup morphogenesis in threedimensional culture. Nature 2011;472:51-56.

32. Lamba DA, Gust J, Reh TA. Transplantation of human embryonic stem cell-derived photoreceptors restores some visual function in Crx-deficient mice. Cell Stem Cell 2009;4:73-79.

33. Kawasaki $\mathrm{H}$, et al. Generation of dopaminergic neurons and pigmented epithelia from primate ES cells by stromal cell-derived inducing activity. Proc Natl Acad Sci U S A 2002;99:1580 1585 .

34. Klimanskaya I, et al. Derivation and comparative assessment of retinal pigment epithelium from human embryonic stem cells using transcriptomics. Cloning Stem Cells 2004;6:217-245.

35. Haruta $\mathrm{M}$, et al. In vitro and in vivo characterization of pigment epithelial cells differentiated from primate embryonic stem cells. Invest Ophthalmol Vis Sci 2004;45:1020-1025.

36. Lu B, et al. Long-term Safety and Function of RPE from Human Embryonic Stem Cells in Preclinical Models of Macular Degeneration. Stem Cells 2009;27:2126-2135.

37. Erdo F, et al. Host-dependent tumorigenesis of embryonic stem cell transplantation in experimental stroke. J Cereb Blood Flow Metab 2003;23:780-785.

38. Tzukerman $M$, et al. An experimental platform for studying growth and invasiveness of tumor cells within teratomas derived from human embryonic stem cells. Proc Natl Acad Sci U S A 2003;100:13507-13512.

39. Temple S. The development of neural stem cells. Nature 2001;414:112-117.

40. Takahashi M, et al. Widespread integration and survival of adultderived neural progenitor cells in the developing optic retina. Mol Cell Neurosci 1998;12:340-348.

41. Young MJ, et al. Neuronal differentiation and morphological integration of hippocampal progenitor cells transplanted to the retina of immature and mature dystrophic rats. Mol Cell Neurosci 2000;16:197-205.

42. Van Hoffelen SJ, et al. Incorporation of murine brain progenitor cells into the developing mammalian retina. Invest Ophthalmol Vis Sci 2003;44:426-434.

43. Nishida A, et al. Incorporation and differentiation of hippocampus-derived neural stem cells transplanted in injured adult rat retina. Invest Ophthalmol Vis Sci 2000;41:4268-4274. 
44. Kurimoto Y, et al. Transplantation of adult rat hippocampusderived neural stem cells into retina injured by transient ischemia. Neurosci Lett 2001;306:57d-60.

45. Francis PJ, et al. Subretinal transplantation of forebrain progenitor cells in nonhuman primates: survival and intact retinal function. Invest Ophthalmol Vis Sci 2009;50:3425-3431.

46. Gamm DM, et al. Protection of visual functions by human neural progenitors in a rat model of retinal disease. PLoS One 2007;2: e338.

47. Wang $\mathrm{S}$, et al. Long-term vision rescue by human neural progenitors in a rat model of photoreceptor degeneration. Invest Ophthalmol Vis Sci 2008;49:3201-3206.

48. Banin E, et al. Retinal incorporation and differentiation of neural precursors derived from human embryonic stem cells. Stem Cells 2006;24:246-257.

49. Lund RD, et al. Human embryonic stem cell-derived cells rescue visual function in dystrophic RCS rats. Cloning Stem Cells 2006;8:189-199.

50. Meyer JS, et al. Embryonic stem cell-derived neural progenitors incorporate into degenerating retina and enhance survival of host photoreceptors. Stem Cells 2006;24:274-283.

51. Arnhold S, et al. Neurally selected embryonic stem cells induce tumor formation after long-term survival following engraftment into the subretinal space. Invest Ophthalmol Vis Sci 2004;45:42514255.

52. Otani A, et al. Bone marrow-derived stem cells target retinal astrocytes and can promote or inhibit retinal angiogenesis. Nat Med 2002;8:1004-1010.

53. Otani A, et al. Rescue of retinal degeneration by intravitreally injected adult bone marrow-derived lineage-negative hematopoietic stem cells. J Clin Invest 2004;114:765-774.

54. Friedlander M, et al. Progenitor cells and retinal angiogenesis. Angiogenesis 2007;10:89-101.

55. Ritter MR, et al. Myeloid progenitors differentiate into microglia and promote vascular repair in a model of ischemic retinopathy. $\mathrm{J}$ Clin Invest 2006;116:3266-3276.

56. Marchetti V, et al. Stemming vision loss with stem cells. J Clin Invest 2010;120:3012-3021.

57. $\mathrm{Lu} \mathrm{B}$, et al. Human adult bone marrow-derived somatic cells rescue vision in a rodent model of retinal degeneration. Exp Eye Res 2010;91:449-455.

58. Tomita $\mathrm{M}$, et al. A comparison of neural differentiation and retinal transplantation with bone marrow-derived cells and retinal progenitor cells. Stem Cells 2006;24:2270-2278.

59. Inoue $\mathrm{Y}$, et al. Subretinal transplantation of bone marrow mesenchymal stem cells delays retinal degeneration in the RCS rat model of retinal degeneration. Exp Eye Res 2007;85:234-241.

60. Tomita $\mathrm{M}$, et al. Bone marrow-derived stem cells can differentiate into retinal cells in injured rat retina. Stem Cells 2002;20:279-283.
61. Arnhold S, et al. Transplantation of bone marrow-derived mesenchymal stem cells rescue photoreceptor cells in the dystrophic retina of the rhodopsin knockout mouse. Graefes Arch Clin Exp Ophthalmol 2007;245:414-422.

62. Wang HC, et al. Transplantation of quantum dot-labelled bone marrow-derived stem cells into the vitreous of mice with laserinduced retinal injury: survival, integration and differentiation. Vision Res 2010;50:665-673.

63. Zhang Y, Wang W. Effects of bone marrow mesenchymal stem cell transplantation on light-damaged retina. Invest Ophthalmol Vis Sci 2010;51:3742-3748.

64. Chaudhry GR, et al. Fate of embryonic stem cell derivatives implanted into the vitreous of a slow retinal degenerative mouse model. Stem Cells Dev 2009;18:247-258.

65. Li L, et al. Human embryonic stem cells possess immuneprivileged properties. Stem Cells 2004;22:448-456.

66. Takahashi K, Yamanaka S. Induction of pluripotent stem cells from mouse embryonic and adult fibroblast cultures by defined factors. Cell 2006;126:663-676.

67. Hirami Y, et al. Generation of retinal cells from mouse and human induced pluripotent stem cells. Neurosci Lett 2009;458:126-131.

68. Meyer JS, et al. Modeling early retinal development with human embryonic and induced pluripotent stem cells. Proc Natl Acad Sci U S A 2009;106:16698-16703.

69. Buchholz DE, et al. Derivation of functional retinal pigmented epithelium from induced pluripotent stem cells. Stem Cells 2009;27:2427-2434.

70. Parameswaran S, et al. Induced pluripotent stem cells generate both retinal ganglion cells and photoreceptors: therapeutic implications in degenerative changes in glaucoma and agerelated macular degeneration. Stem Cells 2010;28:695-703.

71. Lamba DA, et al. Generation, purification and transplantation of photoreceptors derived from human induced pluripotent stem cells. PLoS One 2010;5:e8763.

72. Osakada F, et al. In vitro differentiation of retinal cells from human pluripotent stem cells by small-molecule induction. J Cell Sci 2009;122(pt 17):3169-3179.

73. Carr AJ, et al. Protective effects of human iPS-derived retinal pigment epithelium cell transplantation in the retinal dystrophic rat. PLoS One 2009;4:e8152.

74. Kooreman NG, Wu JC. Tumorigenicity of pluripotent stem cells: biological insights from molecular imaging. J R Soc Interface 2010;7(suppl 6):S753-S763.

75. Gore A, et al. Somatic coding mutations in human induced pluripotent stem cells. Nature 2011;471:63-67.

76. Harness JV, et al. Equivalence of conventionally-derived and parthenote-derived human embryonic stem cells. PLoS One 2011;6:e14499.

77. Lengerke C, Daley GQ. Disease models from pluripotent stem cells. Ann N Y Acad Sci 2009;1176:191-196. 